\title{
Voltammetric Determination of Bergenin with Graphene Modified Glassy Carbon Electrode
}

\author{
Xiaobao Li ${ }^{l}$, Ruyi Zou ${ }^{1}$, Yanyan Niu ${ }^{1}$, Taiming Shao ${ }^{2}$, Yong Chen ${ }^{1}$, Wei Sun ${ }^{1,3^{*}}$, Mengxiong He ${ }^{1}$ \\ ${ }^{1}$ Key Laboratory of Tropical Medicinal Plant Chemistry of Ministry of Education, College of \\ Chemistry and Chemical Engineering, Hainan Normal University, Haikou 571158, P R China \\ ${ }^{2}$ Key Laboratory of Medicinal and Edible Plants Resources of Hainan Province, School of Chemical \\ and Material Engineering, Hainan Institute of Science and Technology, Haikou 571126, P R China \\ ${ }^{3}$ Key Laboratory of Soft Chemistry and Functional Materials of Ministry Education, College of \\ Chemical Engineering, Nanjing University of Science and Technology, Nanjing 210094, P. R. China \\ *E-mail: swyy26@hotmail.com
}

doi: $10.20964 / 2018.02 .68$

Received: 3 October 2017 / Accepted: 14 December 2017 / Published: 28 December 2017

This study utilized a sensitive electroanalytical method for the detection of bergenin in phosphate buffer solution ( $\mathrm{pH}$ 5.0) with a graphene (GR) and chitosan (CS) nanocomposite modified glassy carbon electrode (GCE). Characterization of CS-GR/GCE was carried out by electrochemical impedance spectroscopy. Electrocatalytic oxidation of bergenin on CS-GR/GCE was greatly enhanced with the improvement of the anodic peak current, which allowed the development of a voltammetric sensor for bergenin determination. Under the optimized conditions with differential pulse voltammetry, the anodic peak responses enhanced linearly with bergenin concentration from $2.0 \times 10^{-8} \mathrm{~mol} / \mathrm{L}$ to $8.0 \times 10^{-6} \mathrm{~mol} / \mathrm{L}$ with the detection limit of $3.6 \mathrm{nmol} / \mathrm{L}(3 \sigma)$. This electrochemical sensor showed high sensitivity and selectivity, excellent reproducibility and good stability. The analytical application of this sensor was successfully proved by the Tabellae Bergenini Compositae sample detection.

Keywords: Graphene, Bergenin, Differential pulse voltammetry, Electroanalysis

\section{FULL TEXT}

(C) 2018 The Authors. Published by ESG (www.electrochemsci.org). This article is an open access article distributed under the terms and conditions of the Creative Commons Attribution license (http://creativecommons.org/licenses/by/4.0/). 